\title{
The uterine contraction evaluation is tricky in the in vivo
}

\author{
Hiroyuki Kinoshita $^{1}\left[\right.$. Shinji Kawahito ${ }^{1}$ \\ Received: 4 February 2021 / Accepted: 4 March 2021 / Published online: 9 March 2021 \\ (c) Japanese Society of Anesthesiologists 2021
}

Keywords Contraction · Myometrium · Pressure

To the Editor:

We read the paper by Kimizuka et al. [1] with interest. They have reported the roles of anesthesia-related compounds in the pregnant rat myometrium contraction. We have several concerns, including their methodology. First, Kimizuka et al. [1] adopted a pressure change measured by an intrauterine balloon as a parameter of the in vivo myometrium contraction. However, a balloon pressure change is not always related to uterine contracture, and the method has never been verified as a marker of myometrium contraction. Therefore, they have to prove the accuracy of their study in detail. Second, they did not employ a reference contraction induced by an agent, such as $\mathrm{KCl}$ [2], which has to be used to standardize each force change caused by oxytocin or other anesthesia-related compounds propofol. Alternatively, they may use a set of experiments to verify a time-control contraction of oxytocin to evaluate the intergroup differences [3]. Third, the authors have to explain the discrepancy between their in vitro and in vivo studies in the Discussion section. It must be helpful for the readers to understand the study results. Forth, we advise Kimizuka et al. [1] to use a normoglycemic Krebs solution instead of the high-concentration glucose $(10 \mathrm{mM}=180 \mathrm{mg} / \mathrm{dL})$. Collectively, we would like to await additional studies to verify the conclusion by Kimizuka et al. [1].

\section{References}

1. Kimizuka M, Tokinaga Y, Azumaguchi R, Hamada K, Kazuma S, Yamakage M. Effects of anesthetic agents on contractions of the pregnant rat myometrium in vivo and in vitro. $\mathbf{J}$ Anesth. 2021;35:68-80.

2. Aman M, Hirano K, Nishimura J, Nakano H, Kanaide H. Enhancement of trypsin-induced contraction by in vivo treatment with $17 \mathrm{~B}$-estradiol and progesterone in rat myometrium. Br J Pharmacol. 2005;146:425-34.

3. Jayasooriya GS, Carvalho JCA, Luca A, Balki M. The effects of nitroglycerin on the oxytocin dose-response profile in oxytocindesensitized and Naïve human myometrium: an in vitro study. Anesth Analg. 2021;132:231-9.

Publisher's Note Springer Nature remains neutral with regard to jurisdictional claims in published maps and institutional affiliations.

This comment refers to the article available online at https://doi. org/10.1007/s00540-020-02866-9.

Hiroyuki Kinoshita

hkinoshi@krc.biglobe.ne.jp

1 Department of Anesthesiology, Institute of Biomedical

Sciences, Tokushima University Graduate School, 3-18-15

Kuramoto, Tokushima, Tokushima 770-8503, Japan 